\title{
Optoelectronic sensor of longitudinal and angular displacements
}

\author{
A. Dlugaszek, J. Jabczynski, J. Janucki, W. Skrzeczanowski \\ Institute of Optoelectronics, Military University of Technology, \\ 2 Kaliskiego str. 00-908 Warsaw, POLAND, tel. (48 22) 6859678, fax (48 22) 6668950, \\ e-mail:jabczy@wat.waw.pl,wskrzecz@wat.waw.pl
}

\begin{abstract}
In the sensor a triangulation method of displacement measurement has been used. A method of sensor calibration using interferometric distance measurements has been elaborated. Linearity and resolution investigations have been performed for different parameter sets of sensor transmitting-receiving head. An accuracy of displacement measurements depends on parameters of a beam illuminating a surface being displaced, parameters of a position sensitive detector and on a signal-to-noise ratio of a signal analog processing block. Results of investigations of uncertainties of lathe slide shifts are also presented.
\end{abstract}

Keywords: optoelectronic sensor, position-sensitive detector, longitudial displacement, angular displacement

Paper received 08.09.99; revised manuscript received 30.09.99; accepted for publication 04.10.99.

\section{Introduction}

Measurements of small angular deviations are carried out using interferometry methods as a rule [1]. Such systems (see, for example, [2]) allow displacement measurements only in one plane with $1 \mu \mathrm{rad}$ accuracy. These measurements can be performed also using «geometrical» method, consisting in measurements of $X, Y$ positions of point image in a lens focal plane next divided by focal length $f$.

A triangulation method is one of contactless methods of displacement measurements [3]. In this method a moving object is illuminated by light beam at a certain angle to observation axis. An image of a light spot illuminating moving surface of an object changes its position at position sensitive detector (PSD). A linearized, approximate dependence between displacement $Z$ and a position of a spot image at a detector $X_{A}$ is given by (1):

$$
X_{A} \cong Z \cdot \frac{f_{0}}{Z_{0}-f_{0}} \cdot \sin \alpha,
$$

where: $Z$ is a value of an object displacement from its initial position $Z_{0}, \alpha$ is the angle between light beam illuminating an object and observation object, $f_{0}$ is a focal length of an optical system of a receiving PSD part.

A range of displacement measurement depends on a size of PSD photosensitive area and geometric parameters of optical systems of transmitting and receiving parts of a sen- sor. The range of linearity of dependence (1) is determined by a value of an angle $\alpha$ as well as by readout errors of a position of spot image on PSD surface (nonlinearity of detector characteristics). Next a resolution of displacement measurement depends on signal-to-noise ratio of a signal in a receiving system of a sensor and on resolution of readout system of output electrical signal.

\section{Principle of operation of monolithic PSD detector}

A PSD detector used in a laboratory model is based on monolithic silicon $p-i-n$ photodiode (Fig.1). Upper layer of a doped semiconductor has two deposited readout electrodes. A polarising electrode is deposited on a bottom layer. Light scattered on object surface is collected by optical system and then incidences on detector surface. In the photodiode $i$ layer a conversion of light radiation into electric current flowing from polarising electrode to readout electrodes occurs.Owing to resistance homogeneity of upper layer of a detector, the values of photocurrents reaching readout electrodes are inversely proportional to a distance between place of radiation incidencing on detector surface and a readout electrode (Fig.1). That is why a position of a spot image on PSD surface is given by dependence (2) like that in [2]:

$X_{A}=L / 2 \cdot Y_{n}\left(I_{1}, I_{2}\right)$, 


\section{A. Dlugaszek et al.: Optoelectronic sensor of longitudinal and angular displacements}

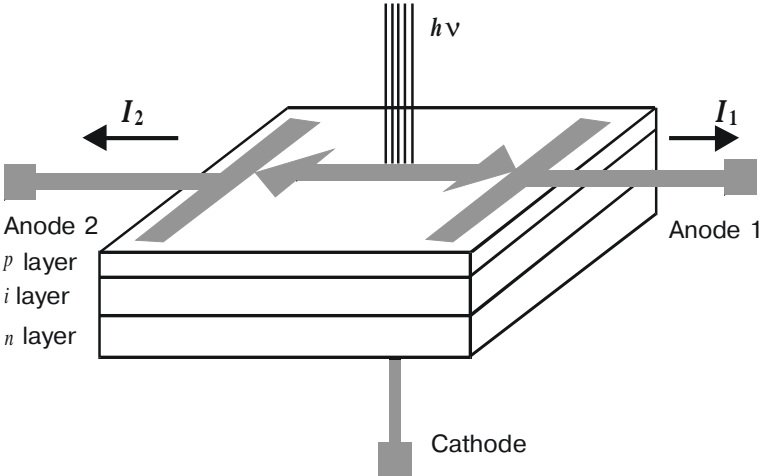

Fig.1. A structure of monolithic PSD detector

where: $X_{A}$ is a spot image position on PSD surface, $I_{1}$ and $I_{2}$ are photocurrents at first and second readout electrodes, respectively, $L$ is a length of photosensitive surface of PSD detector, $Y_{n}$ is a normalising function given by dependence (3):

$Y_{n}(x, y)=\frac{y-x}{y+x}$

Readout resolution of a position of a light spot on detector surface depends on PSD intrinsic noise (thermal noise, shot noise) and on optical noise reaching PSD surface. In order to improve sensor resolution one can use modulation of intensity of a beam illuminating moving object and synchronous reception of radiation scattered on its surface.

Monolithic PSD detectors have many advantages in comparison with such discrete PSD's as CCD [4] or photodiode linear detectors. These are as follows:

- analog, continuous measurement of a position of radiation beam illuminating the surface,

- resolution of position readout,

- high speed of pulse response,

- simple system of electronic processing of a signal.

\section{Sensor calibration}

A calibration of an angular deviation sensor is performed if a value of a coefficient defining a connection between voltage signals from a signal analog processor and angular deviations of radiation beam illuminating PSD surface is found. Measurements have been carried out using laboratory setup shown in Fig.2. He-Ne laser with a UPF beam expander is a radiation source in this set-up.Light beam deviated by means of mirror $\mathrm{M}$ is splitted into two beams in a beam splitter DW. One beam is directed to PSD-2D detection path, while the second one is directed into LBA 100A system for registration of spatial distributions [5]. The use of UFP allows one to achieve a high spatial stability of a light beam. Measurements of spatial stability showed that determining of a beam center position with $1 \mu \mathrm{m}$ accuracy is possible, which for focal length $f=300 \mathrm{~mm}$ gives $3 \mathrm{mrad}$.

Calibrations were based on simultaneous measurements of angular deviations in different directions using LBA 100A system and changes of output voltage signals from the APS processor of the sensor. Calibration curve is shown in Fig.3.
Correlation coefficient $r=0.8$ between LBA measurements and APS voltage signals was found. Mean square deviation of nonlinearity was about $8 \%$.Nonlinearity of a calibration curve, shown in Fig.3, defined as a deviation from the straight line approximating the dependence amounts in this case is $3.4 \%$. The $0.5 \mu \mathrm{m}$ resolution for displacement measurement has been achieved for the measurement range $\Delta Z$. It depends on geometrical parameters of a sensor system, ratio of a light spot diameter to sensor size, and on light and electronic noise. In the case of large displacement range, a measurement nonlinearity (Fig.4) is much higher than in former case and amounts to $24 \%$. It is caused by nonlinearity of the method itself (system responsivity varies according to displacement change from 0.02 up to $0.06 \mathrm{~V} / \mathrm{mm}$ ).

\section{Measurements of angular displacements}

Preliminary measurements of angular displacements have been carried out using arrangement shown in Fig.5. Slide shifts were measured using HP 5526A interferometer. Polarizing beam splitter of the interferometer was attached in a lathe chuck, while prism mirror together with transmitting head GN of the sensor were fasten to the lathe slide. The TD receiving system of a sensor was set on the lathe frame. The stand in Fig.5 allows one to simultaneously measu linearity and angular displacements of lathe slide shift.

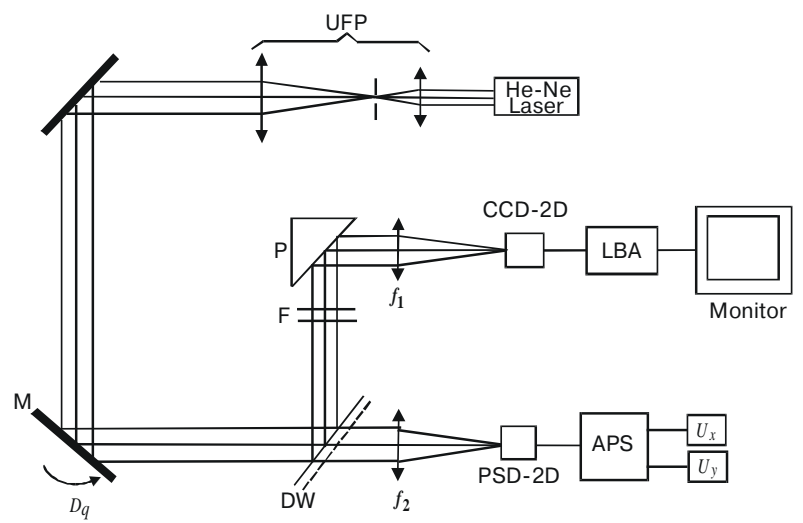

Fig.2. A scheme of a laboratory stand for sensor calibration.

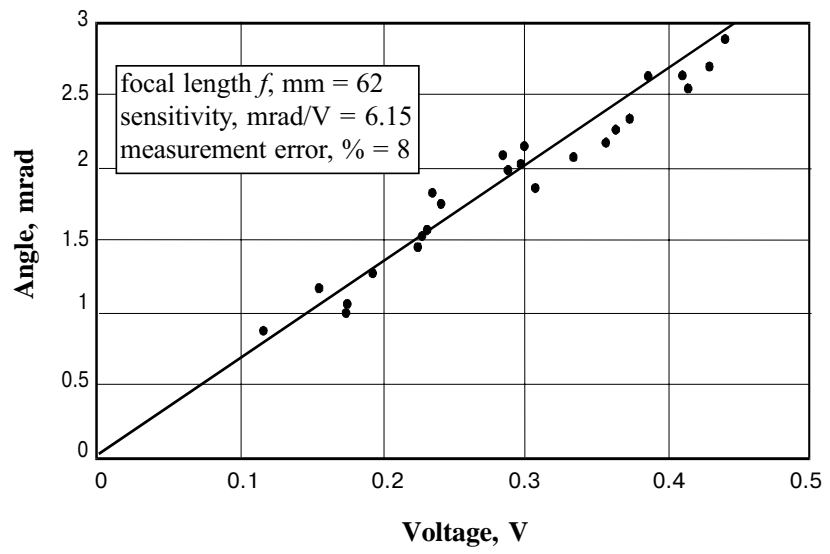

Fig.3. Results of sensor calibration. 


\section{A. Dlugaszek et al.: Optoelectronic sensor of longitudinal and angular displacements}

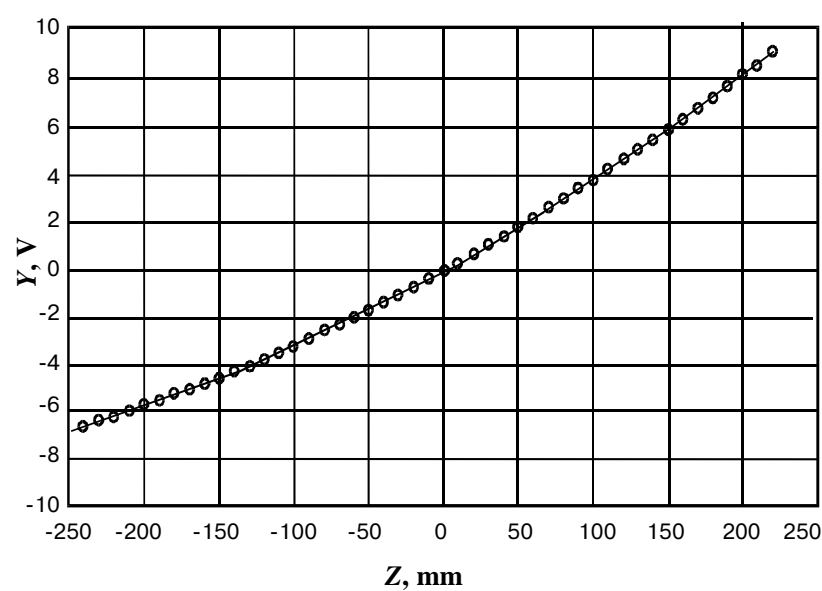

Fig.4. Calibration curve $F=f(Z)$ for the case of large displacement range.

About $2 \mu \mathrm{rad}$ spatial stability of GN beam was found. Both the spatial stability of GN beam and APS resolution effect on the total uncertainty of an angular deviation measurement, which amounted to about $10 \mu \mathrm{rad}$. Measurement of linear slide shift makes a simultaneous calibration of longitudinal lathe shift scale possible. Uncertainty of this calibration is $1 \mu \mathrm{m}$. Results of angular deviation measurements are shown in Fig.6.

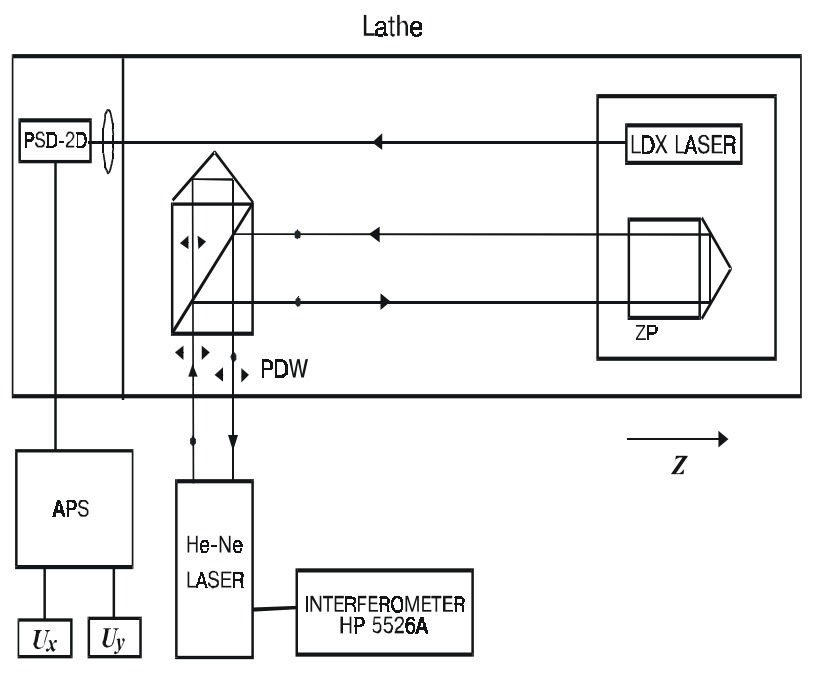

Fig.5. Scheme of a stand for measurements of uncertainties of TUM 25A lathe slide shifts.

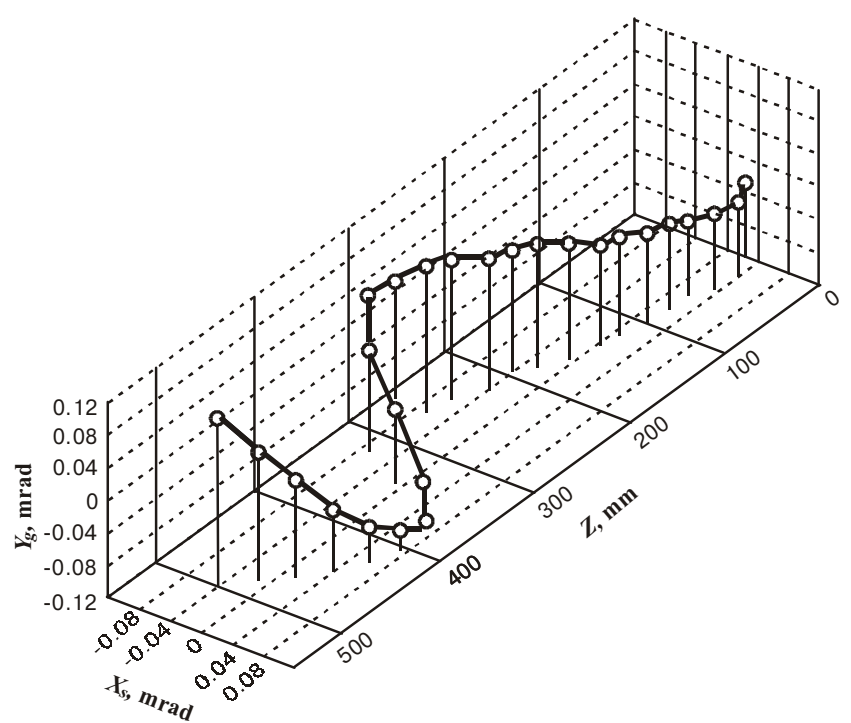

Fig.6. Diagram of angular deviations $Y g$ versus shift $Z$.

\section{Conclusions}

A concept of the sensor of angular deviations was tested in laboratory and tool-room conditions. It seems, that the presented sensor fulfils operational requirements for industrial control instruments. Changes of a focal length in a receiving system of the sensor enable to have different measurement ranges of angular deviations in the investigated object. A high influence of optical noise on measurement resolution is a main disadvantage of the presented version of the sensor. Modulation of GN radiation and synchronous detection of optical signal used for reduction of optical noise should be of destination solution. Application of computer for statistical analysis in real time should ensure further improvement in sensor abilities.

\section{References}

1. K.Holejko, «Interferencyjne metody pomiaru przemieszczen i kata», V Krajowa Szkola Optoelektroniki, Waplewo, 1991, «Metrologia Laserowa», (in Polish), part. II, pp.53-74

2. Hewlett-Packard Co, «Laser Measurement System 5526A»

3. M.Buzinski, A.Levine, W.H.Stevenson, «Laser triangulation range sensors: A study of performance limitations», J.of Las. Appl., pp. 29-36, 1992

4. Hamamatsu Technical Data, «Large Area PSD Series», 1993

5. «Laser Beam Analyzer LBA 100A», Operator's Manual 